OPEN ACCESS

Edited by:

Yu-Xia Chu,

Fudan University, China

Reviewed by:

Wenqiang Cui,

Shandong First Medical

University, China

Yu-Feng Wang,

Harbin Medical University, China

*Correspondence:

Jing Wang

wangjiing1mail@163.com

Specialty section:

This article was submitted to

Musculoskeletal Pain,

a section of the journal

Frontiers in Pain Research

Received: 17 March 2021

Accepted: 11 June 2021

Published: 08 July 2021

Citation:

Wang J, Wang S and Zhang H (2021)

Modulation of Astrocytic Glutamine Synthetase by Endocannabinoid

2-Arachidonoylglycerol in

JNK-Independent Pathway.

Front. Pain Res. 2:682051.

doi: 10.3389/fpain.2021.682051

\section{Modulation of Astrocytic Glutamine Synthetase by Endocannabinoid 2-Arachidonoylglycerol in JNK-Independent Pathway}

\author{
Jing Wang ${ }^{1,2,3 *}$, Shenghong Wang ${ }^{3}$ and Hua Zhang ${ }^{3}$ \\ ${ }^{1}$ School of Clinical Medicine, Southern Medical University, Guangzhou, China, ${ }^{2}$ The People's Hospital of Baoan District, \\ Shenzhen, China, ${ }^{3}$ Department of Orthopaedics, The Affiliated Second Hospital of Lanzhou University, Lanzhou, China
}

Background and Objective: The glutamine synthetase (GS), an astrocyte-specific enzyme, plays an important role in neuroprotection through the glutamate/glutamine shuttle and can be modulated by endocannabinoid (eCB) 2-arachidonoylglycerol (2-AG) through extracellular signal-regulated protein kinase $1 / 2$ (ERK1/2) and p38 signaling pathways. However, the role of c-Jun N-terminal kinase (JNK) signaling pathway in the modulation of GS in astrocytes by 2-AG is not clear.

Materials and Methods: The expression of GS and JNK in astrocytes following the exposure to lipopolysaccharide (LPS) was examined with Western blotting and immunochemistry.

Results: The results revealed that short-term exposure to LPS activated GS and increased phosphorylation of JNK in astrocytes in a time-dependent manner. Treatment with 2-AG reversed the changes in GS but had no effect on the activation of JNK.

Conclusions: These findings suggest that the activation of JNK induced by LPS is not involved in the modulation of astrocytic GS by 2-AG.

Keywords: JNK, cannabinoid receptor, 2-AG, astrocyte, glutamine synthetase

\section{INTRODUCTION}

The astrocytic glutamine synthetase (GS) can modulate the extracellular concentration of glutamate by converting glutamate into glutamine and is verified to be involved in a variety of neurological disorders such as neurodegenerative diseases and chronic pain (1). Endocannabinoids (eCBs) are endogenous mediators of lipid signaling with the capabilities to modulate the synaptic function and to provide neuroprotective and anti-inflammatory effects (2). 2-Arachidonoylglycerol (2-AG) is one of the most abundant $\mathrm{eCBs}$ and plays a potential role in protecting neurocytes from injuries induced by inflammation and insults of neurodegenerative diseases (3). In addition, previous studies found that 2-AG has the capacity of attenuating neuropathic pain and mechanical hyperalgesia in several preclinical models of chronic pain $(4,5)$.

The previous study indicates that $2-\mathrm{AG}$ is involved in the modulation of synaptic function, neuroprotection, and stimulation of mitogen-activated protein kinase (MAPK) family by binding to and activating the G-protein-coupled receptors (GPCR), cannabinoid receptor type $1\left(\mathrm{CB}_{1} \mathrm{R}\right)$, and cannabinoid receptor type $2\left(\mathrm{CB}_{2} \mathrm{R}\right)$, which are expressed in astrocytes $(6,7)$. A variety of 
studies also indicate that the activation of $\mathrm{CB}_{1} \mathrm{R}$ or $\mathrm{CB}_{2} \mathrm{R}$ produced effects of anti-inflammation, antinociception, and neuroprotection (8), and activation of MAPK signaling (9). In addition, activation of $\mathrm{CB}_{1} \mathrm{R}$ or $\mathrm{CB}_{2} \mathrm{R}$ can inhibit the activation of MAPK cascade induced by stress, and the discrepancy remains to be further studied. Our recent study indicates that astrocytic MAPK subunits, extracellular signal-regulated protein kinase $1 / 2(E R K 1 / 2)$ and $\mathrm{p} 38$, are involved in the modulation of GS by $\mathrm{CB}_{1} \mathrm{R}$ and $\mathrm{CB}_{2} \mathrm{R}$ (10). Other studies indicate that $\mathrm{c}$-Jun $\mathrm{N}$-terminal kinase (JNK) participates in the $\mathrm{CB}_{1} \mathrm{R}$-mediated inflammation signaling (11) and $\mathrm{CB}_{2} \mathrm{R}$-mediated suppression of leukocyte migration under inflammation (12). However, there is no study about whether JNK participates in the modulation of astrocytic GS by eCBs. Taking into consideration the importance of cannabinoids (CBs) and astrocytic GS in chronic inflammatory pain, it is necessary to eliminate the role of JNK in astrocytic GS in CB-mediated chronic inflammation, which may be helpful for therapeutic strategy.

\section{MATERIALS AND METHODS}

This study was approved by the Ethics Committees of Animal Usage of Lanzhou University, Southern Medical University, and Shenzhen University.

\section{Primary Cultures of Astrocyte}

The primary culture of astrocytes was performed as described previously (10). In brief, the newborn Sprague-Dawley (SD) rats (postnatal 1-3 days) from the experimental animal center of the Gansu University of Chinese Medicine were decapitated and the cerebral hemispheres were aseptically harvested into Hank's balanced salt solution (HBSS). After the removal of meninges, the cerebral cortices were trimmed into small pieces, followed by digestion with $0.25 \%$ trypsin-ethylenediaminetetraacetic acid (EDTA) (Gibco Life Technology, CA, USA), mechanical dissociation by gentle pipetting with Pasteur pipette, and then centrifugation at $400 \mathrm{~g}$ for $5 \mathrm{~min}$. The cells were resuspended in a culture medium supplemented with $90 \%$ Dulbecco's Modified Eagle Medium/Nutrient Mixture F-12 (DMEM/F12) (Gibco Life Technology, CA, USA) and $10 \%$ fetal bovine serum (FBS; PAN-Biotech, Germany) and plated at a density of 3-5 $\times$ $10^{5}$ cells $/ \mathrm{cm}^{2}$ in $25 \mathrm{~cm}^{2}$ flasks. Cells in flasks were cultured at $37^{\circ} \mathrm{C}$ in a carbon dioxide $\left(\mathrm{CO}_{2}\right)$ incubator for 5-7 days to reach the first confluence. To achieve high pure astrocytes (>95\%), the confluent cells in flasks were shaken at $200 \mathrm{rpm}$ overnight to diminish contamination from microglia. Afterward, the astrocytes were evenly passaged into $35 \mathrm{~mm}$ dishes and treated with $1 \mu \mathrm{g} / \mathrm{ml}$ lipopolysaccharide (LPS), which is one commonly used chemical to induce inflammation and can activate astrocytes via the JNK signaling pathway (13), JNK phosphorylation inhibitor SP600125, or with $0.01 \mu \mathrm{M} 2-\mathrm{AG}$.

Abbreviations: 2-AG, 2-arachidonylglycerol; CBR, cannabinoid receptor; ERK1/2, extracellular signal-regulated protein kinase 1/2; GS, glutamine synthetase; LPS, lipopolysaccharide; MAPK, mitogen-activated protein kinase.

\section{Protein Isolation and Western Blotting}

According to the previous report, the Western blotting was carried out with the manual (10). In brief, astrocytes in $35 \mathrm{~mm}$ dishes were lysed in $100 \mu \mathrm{l}$ radioimmunoprecipitation assay (RIPA) lysis buffer containing $1 \%$ phenylmethanesulfonyl fluoride (PMSF) after different treatments. Lysates were centrifuged at $12,000 \mathrm{rpm}$ for $10 \mathrm{~min}$ to remove cell debris, and the pellet was diluted with $30 \mu \mathrm{l}$ sample buffer. The total protein in lysates was measured for concentration by bicinchoninic acid (BCA) and loaded onto $10 \%$ sodium dodecyl sulfate (SDS)-polyacrylamide gels at 5-20 $\mu \mathrm{g} / \mathrm{lane}$ and then separated by electrophoresis and transferred to polyvinylidene difluoride (PVDF) membranes. Following the non-specific binding sites blockade with $5 \%$ non-fat milk in Tris-buffered saline with Tween-20 (TBST) for $2 \mathrm{~h}$ at room temperature (RT), the PVDF membranes were incubated overnight at $4^{\circ} \mathrm{C}$ with primary antibodies according to the manual of the manufacturer [at a dilution of 1:1,000 for JNK and phospo-JNK (p-JNK) antibodies, \#9252 and \#9255, Cell Signaling Technology, MA, USA; or 1:10,000 for GS antibody; \#ab176562, Abcam, St. Louis, MO, USA] and then washed extensively with TBST three times, $10 \mathrm{~min}$ for each time, and incubated with corresponding secondary antibodies (1:10,000; Cell Signaling Technology, MA, USA) at RT for $2 \mathrm{~h}$. The membranes were then washed three times with TBST at 10-min intervals, and the immunolabeled protein bands on membranes were detected by using an enhanced chemiluminescence kit.

\section{Immunocytochemistry}

After different treatments, the astrocytes cultured on coverslips were fixed with $4 \%$ paraformaldehyde for $30 \mathrm{~min}$ and rinsed with phosphate-buffered saline (PBS). The fixed cells were then permeabilized with $0.4 \%$ Triton X-100 for $20 \mathrm{~min}$, rinsed again with PBS, incubated with $3 \%$ normal goat serum (NGS) for $30 \mathrm{~min}$, and then incubated with different primary antibodies (GS, 1:5,000; JNK and p-JNK, 1:500) overnight at $4{ }^{\circ} \mathrm{C}$, respectively. After $24 \mathrm{~h}$, the coverslips were rinsed with PBS and incubated with corresponding secondary antibodies conjugated with Alexa Fluor ${ }^{\circledR} 488$ (green staining) or 594 (red staining) (Invitrogen, UK) for $2 \mathrm{~h}$ at RT. Then, the coverslips were mounted onto the slide with a mounting medium with 4',6-diamidino-2-phenylindole (DAPI) for the observation of nuclei and sealed with nail gel. The cells were visualized by immunofluorescence microscope (Olympus, Japan).

\section{Statistical Analysis}

All experiments were performed in triplicate and repeated at least three times. STATA software version 14.2 (Stata Corp, College Station, TX, USA) was used for statistical analysis, and the data were expressed as the mean \pm SEM. One-way ANOVA followed by the Newman-Keuls test was used to assess the significant differences, and $p<0.05$ was considered as significantly different. 


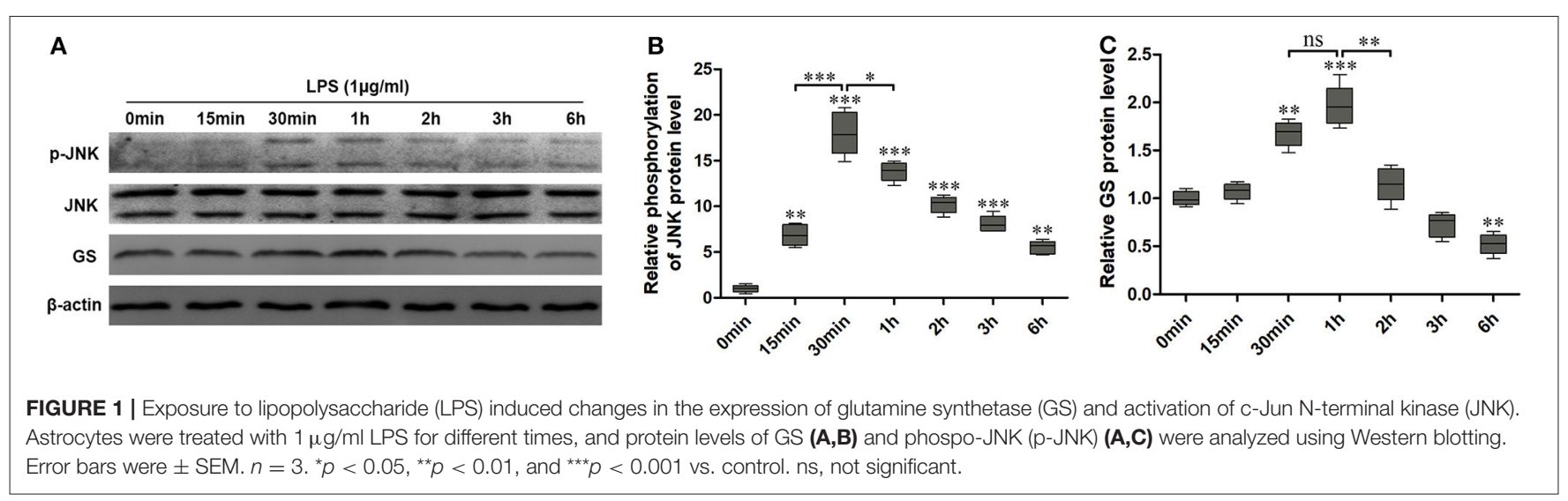

\section{RESULTS}

\section{Lipopolysaccharide (LPS) Activated Expression of GS and Phosphorylation of JNK and Translocation in Primary Astrocytes of Rats}

Similar to our previous study (10), $1 \mu \mathrm{g} / \mathrm{ml}$ LPS was used to activate the astrocytes. To investigate the effects of LPS on the JNK pathway and expression of GS in astrocytes, the expressions of $\mathrm{p}-\mathrm{JNK}, \mathrm{JNK}$, and GS in primary astrocyte culture were evaluated using Western blotting after treatment with DMEM/F12 containing $1 \mu \mathrm{g} / \mathrm{ml}$ LPS at $0 \mathrm{~min}, 15 \mathrm{~min}, 30 \mathrm{~min}$, $1 \mathrm{~h}, 2 \mathrm{~h}, 3 \mathrm{~h}$, and $6 \mathrm{~h}$. The data showed that the exposure to LPS induced time-dependent biphasic changes in the expression of GS in astrocytes, i.e., in contrast to baseline ( $0 \mathrm{~min})$, expression of GS began to increase at $30 \mathrm{~min}(1.62 \pm 0.08, p<0.01)$, peaked at $1 \mathrm{~h}(1.86 \pm 0.08, p<0.001)$, declined to control level at $2-3 \mathrm{~h}$, and then decreased at $6 \mathrm{~h}(0.57 \pm 0.05, p<0.01$; Figures 1A,B $)$. Regarding the JNK pathway, LPS significantly increased the phosphorylation of JNK in a time-dependent manner while without effect on the total JNK. The protein level of p-JNK increased at $15 \mathrm{~min}(6.96 \pm 0.63, p<0.01)$, reached a maximal level at $30 \mathrm{~min}(18.12 \pm 0.90, p<0.001)$, and then gradually declined but was still higher at $6 \mathrm{~h}(5.60 \pm 0.49, p<0.01)$ than control (Figures 1A,C). In addition, previous studies indicated that the JNK pathway exerted its role through translocation from the cytoplasm to nucleus (14). As expected, $1 \mu \mathrm{g} / \mathrm{ml}$ LPS for 1 $\mathrm{h}$ exposure induced the translocation of JNK from cytoplasm to nucleus, which was prevented by JNK phosphorylation inhibitor SP600125 (Figure 2). In addition, SP600125 also prevented the expression of GS by LPS.

\section{2-Arachidonoylglycerol (2-AG) Reversed Changes in LPS-Induced GS Independently on the p-JNK Pathway}

To explore the effects of $2-\mathrm{AG}$ on the activation of astrocytes induced by exposure to LPS, the astrocytes were exposed to $1 \mu \mathrm{g} / \mathrm{ml} \mathrm{LPS}$ for $1 \mathrm{~h}$ and were chosen on the basis of the acquired data from Figure 1. The cells were pretreated with $1 \mu \mathrm{M} 2-\mathrm{AG}$ for
$1 \mathrm{~h}$ and/or $1 \mu \mathrm{g} / \mathrm{ml}$ LPS for $1 \mathrm{~h}$. Compared with control $(0 \mu \mathrm{g} / \mathrm{ml}$ LPS), exposure of astrocytes to $1 \mu \mathrm{g} / \mathrm{ml} \mathrm{LPS}$ for $1 \mathrm{~h}$ significantly elevated the expressions of p-JNK $(2.18 \pm 0.18, p<0.01)$ and GS $(3.41 \pm 0.29, p<0.01)$ and treatment with $2-\mathrm{AG}$ could significantly reverse the changes in the expression of GS induced by exposure to LPS $(1.13 \pm 0.09, p<0.01)$ when compared with LPS group (Figures 3A,B) but without significant effect on the expression of p-JNK (Figures 3A,C).

\section{Dephosphorylation of JNK Increased the Expression Level of GS}

According to the above results, phosphorylation of JNK is not the pathway of 2-AG modulating the expression of GS in astrocytes. To further address the question of whether the JNK pathway was involved in the process of regulating the expression of GS, a specific inhibitor for the JNK signaling pathway, SP600125, was used to investigate the relationship between the JNK pathway and the expression of GS in astrocytes. SP600125 at the concentration of $50 \mu \mathrm{M}$ and $100 \mu \mathrm{M}$ could significantly inhibit LPS-induced activation of JNK in a dose-dependent manner (16.71 \pm 0.75 , $p<0.05 ; 5.03 \pm 0.33, p<0.001)$ when compared with LPS alone (22.41 \pm 1.18 ) (Figures 4A,C). Meanwhile, SP600125 at the concentration of 50 and $100 \mu \mathrm{M}$ could also significantly suppress the LPS-induced upregulation of expression of GS in a dosedependent manner $(1.15 \pm 0.84, p<0.01 ; 0.79 \pm 0.06, p<0.01)$ when compared with LPS alone $(2.14 \pm 0.15)$ (Figures 4A,B). In addition, SP600125 also inhibited the translocation of $\mathrm{p}$ JNK (Figure 2). Briefly, these data suggested that LPS activated JNK resulting in the upregulation of expression of GS. In the other words, GS was the downstream target of JNK signaling in astrocytes.

\section{DISCUSSION}

Previous studies indicate that GS is involved in suppressing the development of glutamate/ammonia neurotoxicity and a variety of neurological diseases, such as neuropathic pain and inflammatory pain (1). Intriguingly, both the increase and the decrease of GS are reported in the same diseases, such as hepatic encephalopathy, traumatic brain injury, and epilepsy, but 
contrarily, controlling the expression of GS can diminish these diseases. Consistent with our previous study (10), this study finds that exposure to LPS resulted in the expression of GS in a biphasic form in astrocytes, i.e., the expression of GS is increased with short-term exposure to LPS and decreased with long-term exposure to LPS.

The MAPK family of kinases including p38 and ERK participate in pain and neurodegenerative diseases and exist in activated astrocytes induced by pathological stimulation (6). Our previous study indicates that exposure to LPS could activate p38 and ERK1/2 in astrocytes with different patterns (10). In this study, we find that inhibition of JNK blocks the increase in GS

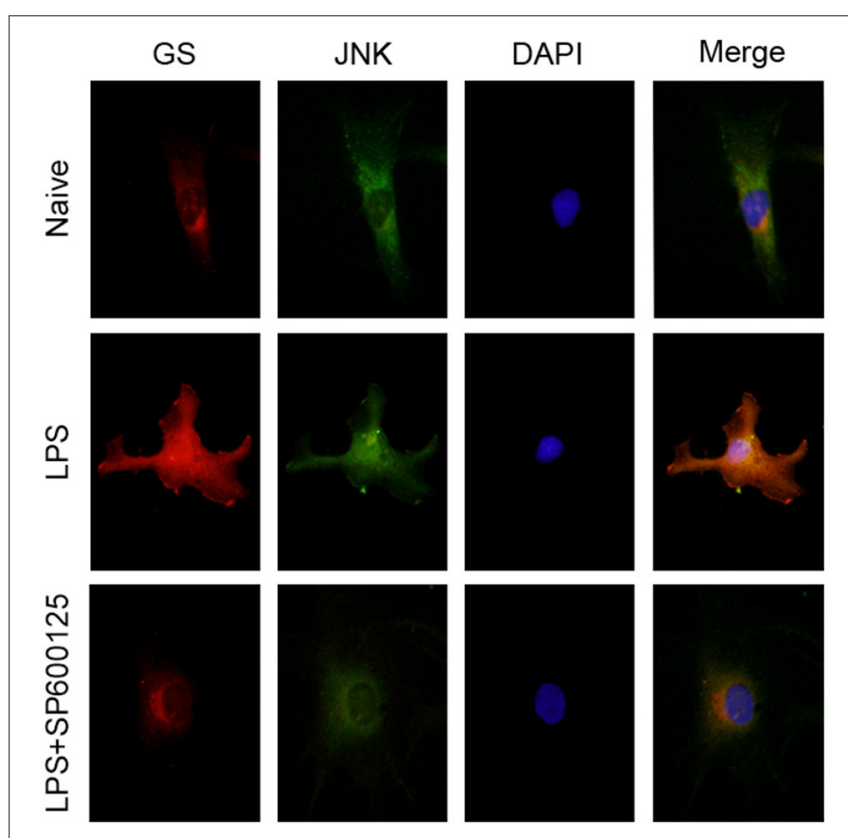

FIGURE 2 | Inhibition of phosphorylation of JNK prevented the translocation of JNK and upregulation of GS induced by exposure to LPS. Astrocytes were pretreated with SP600125 for $1 \mathrm{~h}$ and exposed to $1 \mu \mathrm{g} / \mathrm{ml}$ LPS for $1 \mathrm{~h}$. Immunocytochemistry assay was used to analyze the translocation of JNK and changes in the expression of GS. Scar bar $=10 \mu \mathrm{m}$ by LPS, of which the mechanism may be through suppression of glucocorticoid receptor transcriptional activity (15). This study further indicates that exposure to LPS produces a uniphasic activation of JNK in astrocytes, which enriches the involvement of MAPK signaling in LPS-induced changes in GS in astrocytes. However, the mechanism remains to be further studied.

2-Arachidonoylglycerol (2-AG) is an eCB that binds to $\mathrm{CB}_{1} \mathrm{R}$ and $\mathrm{CB}_{2} \mathrm{R}$ expressed in astrocytes. The previous study found that astrocytic p38 can be activated by $2-\mathrm{AG}$, while blocking $\mathrm{CB}_{1} \mathrm{R}$ can produce an inhibitory effect on the modulation of 2-AG on p38 (9), implying that 2-AG participates in the modulation of MAPK signaling in astrocytes. Our previous study suggests that, under the condition of short-term exposure to LPS, activation of p38 could increase the expression of GS, and 2AG could suppress the increased expression of GS by inhibiting the phosphorylation level of p38. While under the condition of long-term exposure to LPS, activation of ERK1/2 results in a decrease of expression of GS and 2-AG reverses the decrease of expression of GS through reducing the activation of ERK1/2 (10). It should be noted, in our previous study, that although ERK1/2 and p38 are activated by short-term exposure to LPS and longterm exposure to LPS, respectively, the activation is relatively weaker compared to long-term and short-term exposure to LPS, respectively. This study indicates that JNK is activated during the short-term exposure to LPS, while 2-AG has no effect on the phosphorylation of JNK. However, other studies indicate that JNKs are involved in the $\mathrm{CB}_{1} \mathrm{R}$-mediated inflammation signaling (11) and $\mathrm{CB}_{2} \mathrm{R}$-mediated suppression of leukocyte migration under inflammation (12). These results imply that activation of JNK may be the upstream pathway of the astrocytic extracellular matrix (ECM) system in modulating inflammation, which remains to be further investigated.

In conclusion, this study indicated that exposure to LPS for the short term and long term can produce different changes in the activities of GS in astrocytes with activation of JNK. ECB 2AG modulates the expression of GS induced by exposure to LPS, which is not dependent on the activation of JNK. The mechanism of JNK in modulating the astrocytic expression of GS remains to be further studied.

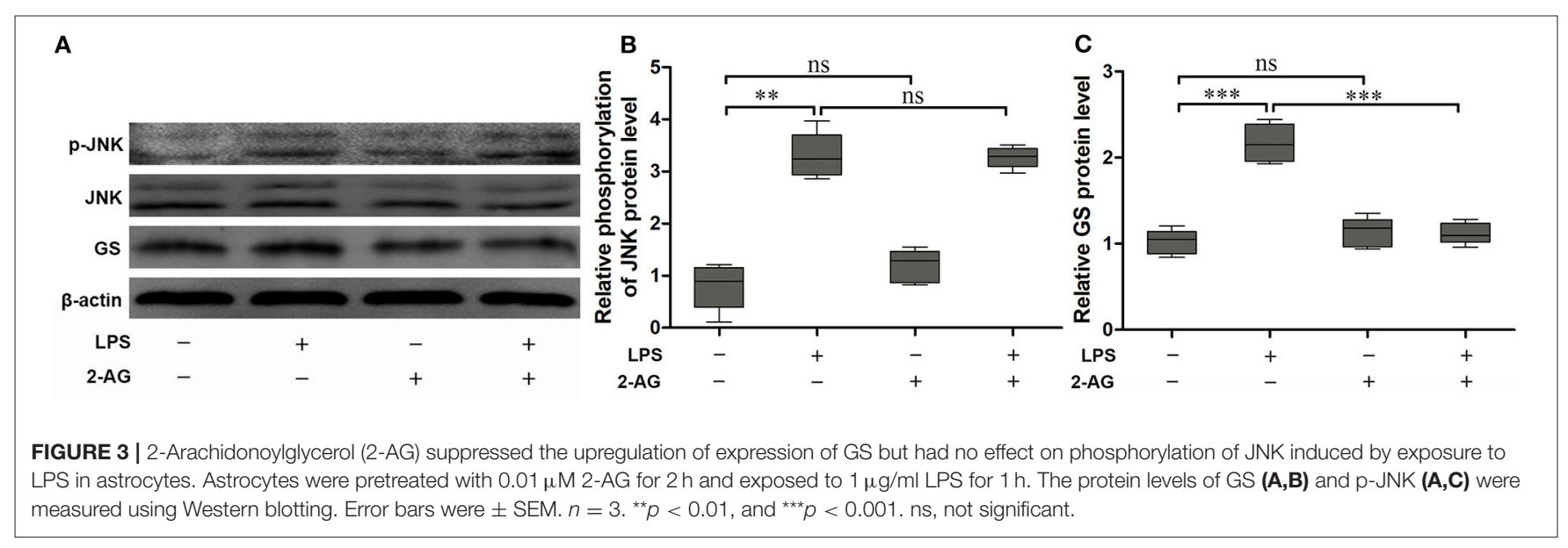






\section{DATA AVAILABILITY STATEMENT}

The original contributions presented in the study are included in the article/supplementary material, further inquiries can be directed to the corresponding author/s.

\section{ETHICS STATEMENT}

The animal study was reviewed and approved by the Animal Ethic Committee of Lanzhou University.

\section{CONSENT FOR PUBLICATION}

The authors all consented to publication.

\section{REFERENCES}

1. Mostafeezur RM, Shinoda M, Unno S, Zakir HM, Takatsuji H, Takahashi K, et al. Involvement of astroglial glutamate-glutamine shuttle in modulation of the jaw-opening reflex following infraorbital nerve injury. Eur J Neurosci. (2014) 39:2050-9. doi: 10.1111/ejn.12562

2. Marrs WR, Blankman JL, Horne EA, Thomazeau A, Lin YH, Coy J, et al. The serine hydrolase ABHD6 controls the accumulation and efficacy of 2-AG at cannabinoid receptors. Nat Neurosci. (2010) 13:951-7. doi: 10.1038/nn.2601

3. Zhang J, Chen C. Endocannabinoid 2-arachidonoylglycerol protects neurons by limiting COX-2 elevation. J Biol Chem. (2008) 283:22601-11. doi: 10.1074/jbc.M800524200

4. Costa B1, Colleoni M, Conti S, Parolaro D, Franke C, Trovato AE, et al. Oral anti-inflammatory activity of cannabidiol, a non-psychoactive constituent of cannabis, in acute carrageenan-induced inflammation in the rat paw. Naunyn Schmiedebergs Arch Pharmacol. (2004) 369:2949. doi: 10.1007/s00210-004-0871-3

5. Khasabova IA, Yao X, Paz J, Lewandowski CT, Lindberg AE, Coicou L, et al. JZL184 is anti-hyperalgesic in a murine model of cisplatin-induced peripheral neuropathy. Pharmacol Res. (2014) 90:67-75. doi: 10.1016/j.phrs.2014.09.008

6. Huang W, Lv B, Zeng H, Shi D, Liu Y, Chen F, et al. Paracrine factors secreted by MSCs promote astrocyte survival associated With GFAP downregulation after ischemic stroke via p38 MAPK and JNK. J Cell Physiol. (2015) 230:246175. doi: $10.1002 /$ jcp. 24981

7. Pellkofer HL, Havla J, Hauer D, Schelling G, Azad SC, Kuempfel T, et al. The major brain endocannabinoid 2-AG controls neuropathic pain and mechanical hyperalgesia in patients with neuromyelitis optica. PLoS ONE. (2013) 8:e71500. doi: 10.1371/journal.pone.0071500

\section{AUTHOR CONTRIBUTIONS}

SW performed the experiments and analyzed the data. HZ analyzed the data. JW designed the experiments and wrote the manuscript. All authors contributed to the article and approved the submitted version.

\section{ACKNOWLEDGMENTS}

The authors thank the support from the National Natural Science Foundation of China (Grant No. 81371230) and the Basic Research Foundation of Medical and Health care of Bao'an Health and Family Planning Commission (No. 2019JD044).

8. Molina-Holgado F, Molina-Holgado E, Guaza C, Rothwell NJ. Role of $\mathrm{CB} 1$ and $\mathrm{CB} 2$ receptors in the inhibitory effects of cannabinoids on lipopolysaccharide-induced nitric oxide release in astrocyte cultures. $J$ Neurosci Res. (2002) 67:829-36. doi: 10.1002/jnr.10165

9. Derkinderen P, Ledent C, Parmentier M, Girault JA. Cannabinoids activate p38 mitogen-activated protein kinases through CB1 receptors in hippocampus. J Neurochem. (2001) 77:95760. doi: 10.1046/j.1471-4159.2001.00333.x

10. Wang S, Zhang H, Geng B, Xie Q, Li W, Deng Y, et al. 2-arachidonyl glycerol modulates astrocytic glutamine synthetase via $\mathrm{p} 38$ and ERK1/2 pathways. $J$ Neuroinflammation. (2018) 15:220. doi: 10.1186/s12974-018-1254-X

11. Yan W, Cao Y, Yang H, Han N, Zhu X, Fan Z, et al. CB1 enhanced the osteo/dentinogenic differentiation ability of periodontal ligament stem cells via p38 MAPK and JNK in an inflammatory environment. Cell Prolif. (2019) 52:e12691. doi: 10.1111/cpr.12691

12. Liu YJ, Fan HB, Jin Y, Ren CG, Jia XE, Wang L, et al. Cannabinoid receptor 2 suppresses leukocyte inflammatory migration by modulating the JNK/c-Jun/Alox5 pathway. J Biol Chem. (2013) 288:13551-62. doi: 10.1074/jbc.M113.453811

13. Liu X, Cheng C, Shao B, Wu X, Ji Y, Liu Y, et al. CDK11(p58) promotes rat astrocyte inflammatory response via activating p38 and JNK pathways induced by lipopolysaccharide. Neurochem Res. (2012) 37:563-73. doi: 10.1007/s11064-011-0 643-7

14. Chang L, Karin M. Mammalian MAP kinase signalling cascades. Nature. (2001) 410:37-40. doi: 10.1038/35065000

15. Oren A, Herschkovitz A, Ben-Dror I, Holdengreber V, Ben-Shaul Y, Seger R, et al. The cytoskeletal network controls c-Jun expression and glucocorticoid 
receptor transcriptional activity in an antagonistic and cell-type-specific manner. Mol Cell Biol. (1999) 19:1742-50. doi: 10.1128/MCB.19.3.1742

Conflict of Interest: The authors declare that the research was conducted in the absence of any commercial or financial relationships that could be construed as a potential conflict of interest.
Copyright $\odot 2021$ Wang, Wang and Zhang. This is an open-access article distributed under the terms of the Creative Commons Attribution License (CC BY). The use, distribution or reproduction in other forums is permitted, provided the original author(s) and the copyright owner(s) are credited and that the original publication in this journal is cited, in accordance with accepted academic practice. No use, distribution or reproduction is permitted which does not comply with these terms. 